\title{
All-optical endoscopic probe for high resolution 3D photoacoustic tomography
}

\author{
R. Ansari, E. Zhang, A. E. Desjardins, and P. C. Beard \\ Department of Medical Physics and Biomedical Engineering, University College London, \\ Gower Street, WC1E 6BT, UK
}

\begin{abstract}
A novel all-optical forward-viewing photoacoustic probe using a flexible coherent fibre-optic bundle and a FabryPérot (FP) ultrasound sensor has been developed. The fibre bundle, along with the FP sensor at its distal end, synthesizes a high density 2D array of wideband ultrasound detectors. Photoacoustic waves arriving at the sensor are spatially mapped by optically scanning the proximal end face of the bundle in $2 \mathrm{D}$ with a $\mathrm{CW}$ wavelength-tunable interrogation laser. 3D images are formed from the detected signals using a time-reversal image reconstruction algorithm. The system has been characterized in terms of its PSF, noise-equivalent pressure and field of view. Finally, the high resolution 3D imaging capability has been demonstrated using arbitrary shaped phantoms and duck embryo.
\end{abstract}

Keywords: Photoacoustic tomography, photoacoustic endoscopy, Coherent fibre bundle, Image bundle, FabryPérot sensors.

\section{INTRODUCTION}

Photoacoustic endoscopy (PAE) has many potential clinical imaging applications such as guiding interventional procedures in fetal medicine, and assessing the gastrointestinal tract and other hollow organs. ${ }^{1-5}$ Most previous PAE probes employ mechanically scanned piezoelectric transducers at the distal end. In this study, we demonstrate the feasibility of a novel, all-optical forward-viewing endoscopic probe that uses a flexible coherent fibre bundle to optically address different spatial points on a Fabry-Pérot (FP) ultrasound sensor. This method avoids the need for mechanical scanning at the distal end of the probe, and the FP sensor offers advantages in terms of ease of fabrication and wideband acoustic performance over piezoelectric equivalents. ${ }^{6-9}$ Moreover, because the FP sensor is transparent, a forward viewing configuration can readily be achieved. By contrast, it is challenging to achieve a forward viewing configuration with sufficient miniaturization using opaque piezoelectric receivers due to the need for the detector to be laterally offset from the excitation laser beam.

\section{EXPERIMENTAL SET-UP}

To demonstrate the concept, the experimental set-up shown in Figure 1 was used. The system comprises a commercially available flexible coherent fibre bundle and a free-space Fabry-Pérot (FP) ultrasound sensor. ${ }^{10,11}$ The fibre bundle has approximately 18,000 step-index fibre-optic cores (0.8 NA; $10 \mu \mathrm{m}$ core diameter) with an active diameter of $1.25 \mathrm{~mm}$ (outer diameter is $1.5 \mathrm{~mm}$ ). The FP sensor was formed onto a PMMA substrate by depositing a $15 \mu \mathrm{m}$ thick polymer spacer layer (Parylene C) sandwiched between two dielectric mirrors coatings, which were designed to be reflective to the interrogation wavelengths and transmissive to the excitation wavelengths for backward-mode photoacoustic imaging. A wavelength-tunable (400 to $2100 \mathrm{~nm}$ ) optical parametric oscillator (OPO) based laser system was used as an excitation source, which provided 8 ns duration pulses at a $30 \mathrm{~Hz}$ pulse repetition rate. For demonstration purposes, the excitation light was coupled via a dichroic mirror at the distal end and transmitted through the sensor and into the sample; in a practical endoscopic implementation, however, it is anticipated that the excitation light would be delivered through the bundle. Using the experimental setup shown in Figure 1, the proximal end of the bundle was optically scanned with a CW wavelength-tunable interrogation laser beam (1550 nm center wavelength) in order to address different spatial points on the FP sensor located at the distal end. The distal end face of the fibre bundle was imaged onto the FP ultrasound sensor using a free-space 1:4 optical relay to increase the imaging aperture to $5 \mathrm{~mm}$. The photoacoustic signals are mapped by sequentially interrogating the FP sensor through the fibre-optic cores. Because the fibre-optic cores

Photons Plus Ultrasound: Imaging and Sensing 2017, edited by Alexander A. Oraevsky, Lihong V. Wang, Proc. of SPIE Vol. 10064, 100641W · C 2017 SPIE · CCC code: 1605-7422/17/\$18 · doi: 10.1117/12.2250617 


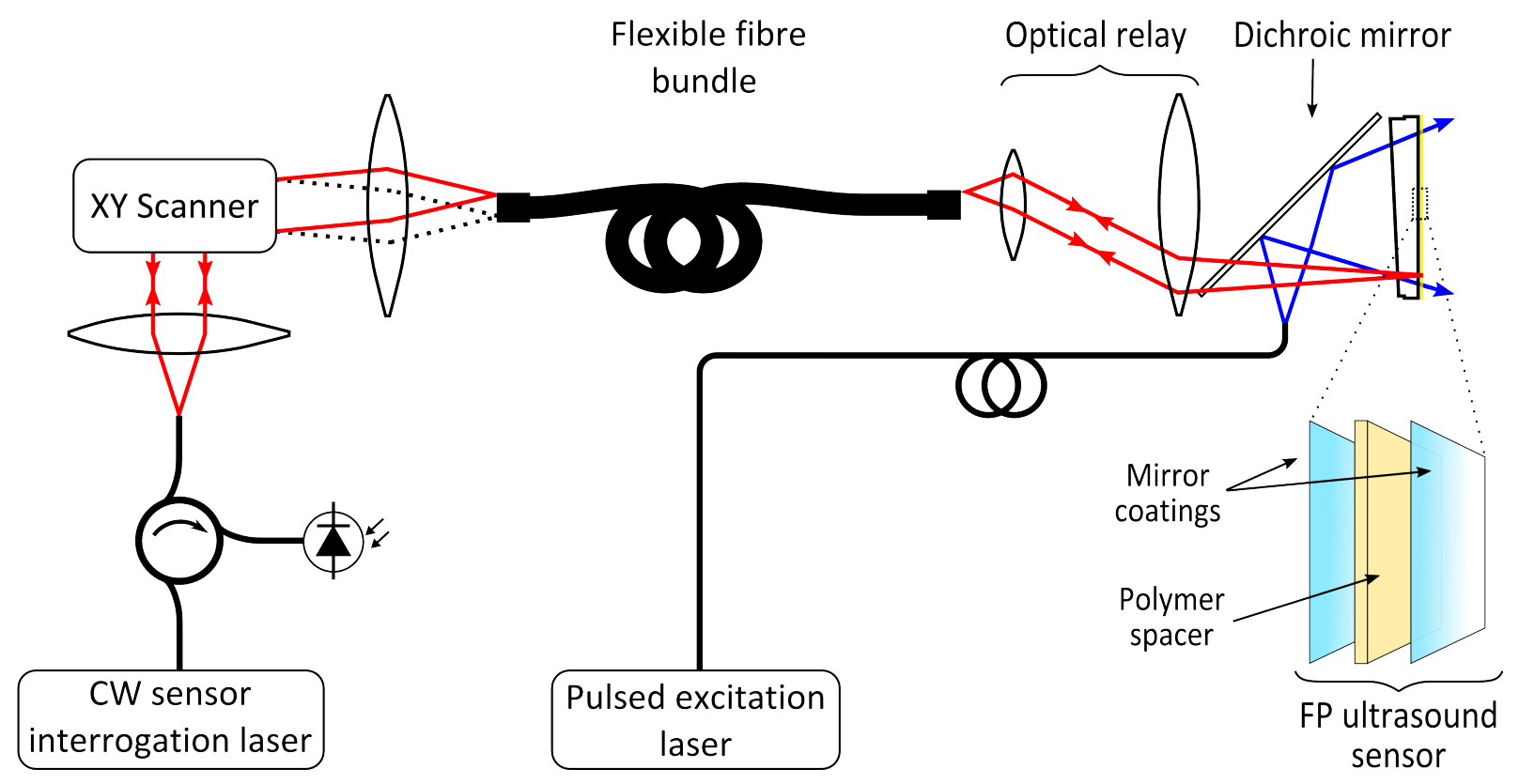

Figure 1. Experimental set-up of the flexible photoacoustic endoscopy (PAE) system. The close-up shows different layers that form the Fabry-Pérot ultrasound sensor.

are arranged in a hexagonal pattern, the raw PA signals are interpolated onto a uniform rectilinear grid before reconstructing the PA images.

\section{RESULTS AND DISCUSSION}
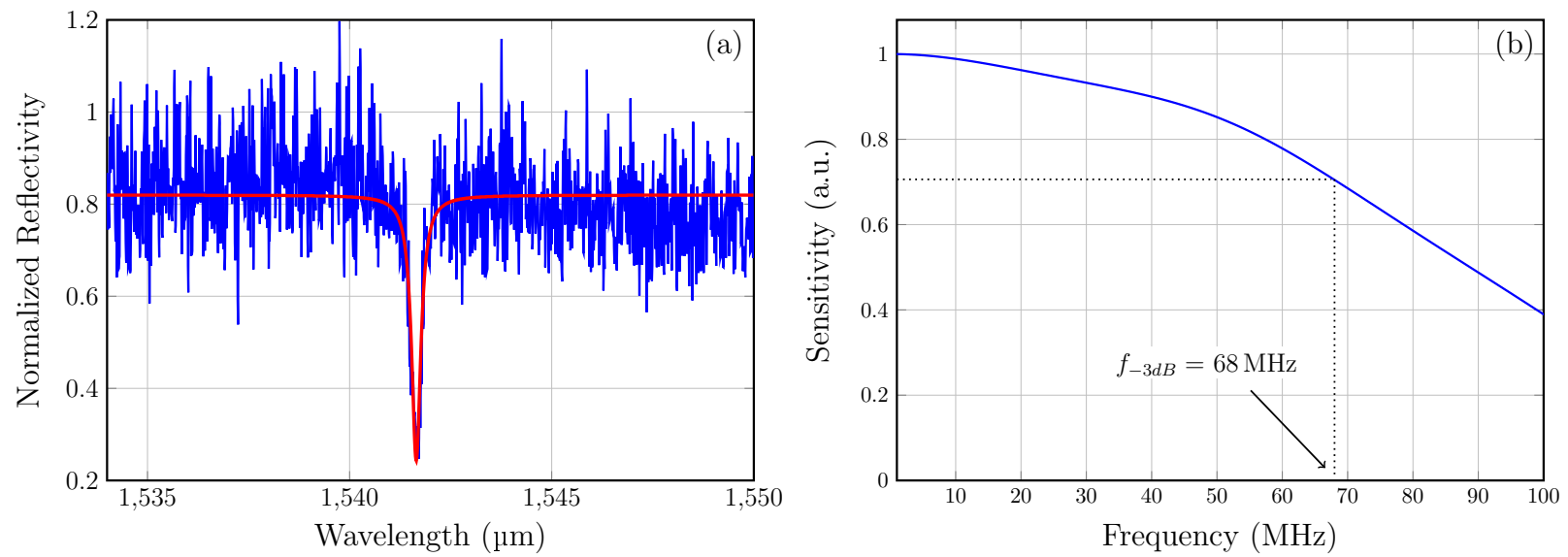

Figure 2. (a) Interferometer transfer function (ITF) of the FP ultrasound sensor located at the distal end of the flexible PAE probe (blue), and its Lorentzian fit (red). (b) Simulated frequency response of the 15 pm thick FP ultrasound sensor which has an acoustic bandwidth $(-3 \mathrm{~dB})$ of $68 \mathrm{MHz}{ }^{6,12}$

The interferometer transfer function (ITF) of the Fabry-Pérot sensor measured through a single fibre-optic core is shown in Figure 3(a) (blue). In addition to the primary fringe from the FP cavity, the ITF also exhibits high frequency oscillations which are caused by optical crosstalk between adjacent cores. This is a consequence of the very tightly packed cores in the common cladding material, which results in wavelength dependent coupling between adjacent cores. An asymmetric Lorentzian function was fitted (red trace in Figure 3(a)) to the ITF 
to determine the optimum bias wavelength (the wavelength that corresponds to the maximum slope of the ITF and yields maximum sensitivity). Figure 3(b) shows the simulated frequency response of the $15 \mu \mathrm{m} \mathrm{FP}$ sensor, which provides a detection bandwidth $(-3 \mathrm{~dB})$ of $68 \mathrm{MHz} .{ }^{12}$ The noise equivalent pressure (NEP) of the sensor was $300 \mathrm{~Pa}$ for a measurement bandwidth of $20 \mathrm{MHz}$.

\subsection{Spatial resolution}

The spatial resolution of the system was evaluated by imaging rows of absorbing ribbons that provide a step edge in the lateral direction. The schematic in Figure 3(a) shows arrangement of the ribbons with respect to the PAE probe. Deionized water was used to provide acoustic coupling between the FP sensor and the ribbon phantom. PA signals were acquired using an excitation wavelength of $1064 \mathrm{~nm}$ and a fluence of $20 \mathrm{~mJ} \mathrm{~cm}^{-2}$. The interrogation beam was scanned in 2D over the proximal face of the bundle in order map the spatial distribution of the PA waves incident on the FP sensor at the distal end; Figure 3(b) shows the detected PA signals P(x, t) along a line perpendicular to the ribbon length. A 3D PA image was reconstructed using a time-reversal algorithm. ${ }^{13}$ Figure 3(c) shows an $\mathrm{x}-\mathrm{z}$ slice from the reconstructed PA image illustrating that the ribbon cross-sections are clearly visualized up to a depth of $7 \mathrm{~mm}$.

The spatial resolution of the PAE probe was determined by taking lateral and axial profiles (Figure 3(e,f)) through the reconstructed ribbon feature shown in Figure 3(d). The lateral spatial resolution at a depth of $1 \mathrm{~mm}$ is $57 \mu \mathrm{m}$, which was calculated by fitting a sigmoidal curve to the lateral profile and taking the FWHM width of its derivative (Figure 3(e)). The axial line spread function is $46 \mu \mathrm{m}$, which was determined by taking the FWHM width of a profile in the z-direction as shown in Figure 3(f). This is likely to be an underestimate of the axial resolution, because the ribbons were thicker than the one half of the wavelength that corresponds to the maximum detectable frequency.
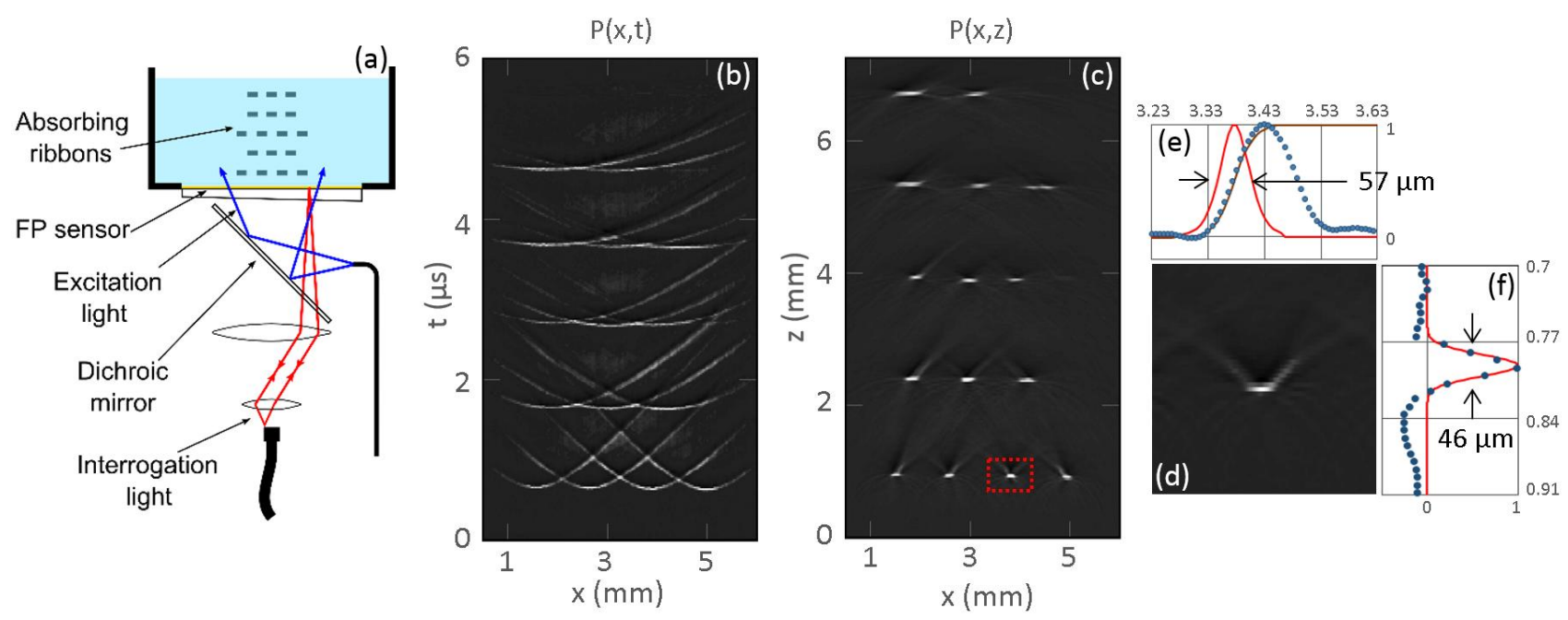

Figure 3. : Spatial resolution of the flexible PAE probe in tomography mode. (a) Experimental setup shows the arrangement of ribbon phantom with respect to the PAE probe. (b) Raw photoacoustic signals from the ribbon phantom from a plane perpendicular to the ribbon length. (c) Reconstructed PA image that shows cross-section of the ribbons at different depths. (d) Zoomed in image of the feature highlighted in (c). (e) Lateral profile through the feature in (d) and its edge response. (f) Axial profile through the feature in (d) and its Gaussian fit.

\subsection{Arbitrary shaped phantoms}

The three-dimensional imaging capability of the system was demonstrated by imaging arbitrary shaped phantoms. The top panel in Figure 4 shows the widefield microscope images of a synthetic hair knot, twisted ribbons, and a leaf skeleton phantom coated in India ink. These phantoms were immersed in a deionized water bath (similar to the setup shown in Figure 3(a)) and PA signals were acquired using a $1064 \mathrm{~nm}$ excitation wavelength and a 

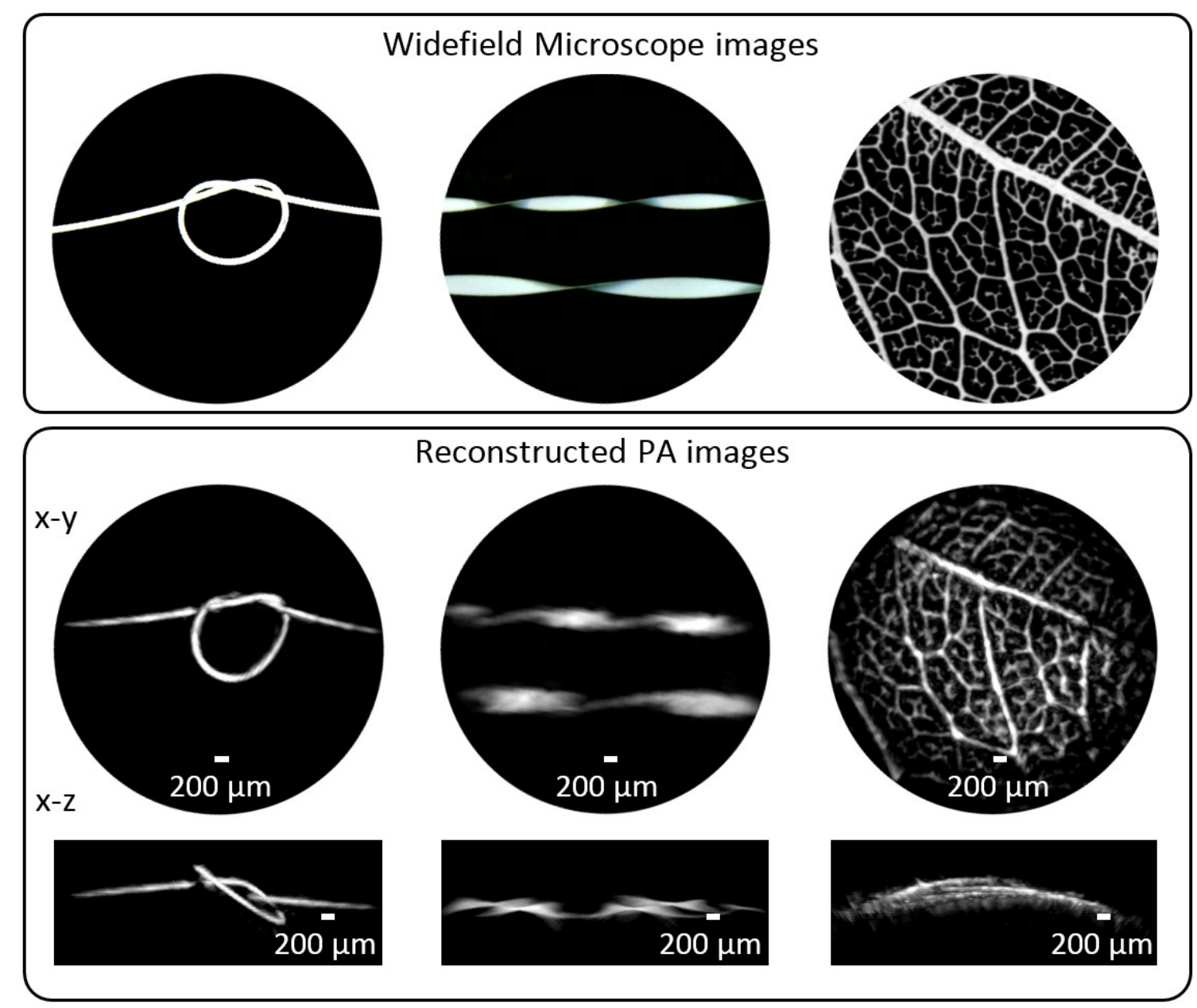

Figure 4. PA images of the arbitrary shaped phantoms acquired using an excitation wavelength of $1064 \mathrm{~nm}$ and a fluence of $20 \mathrm{~mJ} \mathrm{~cm}^{-2}$. (top panel) Widefield microscope images of a synthetic hair knot, twisted ribbons, and a leaf skeleton phantom coated in India ink. (bottom panel) Reconstructed PA images of the phantoms shown in top panel, maximum intensity projected along the $\mathrm{x}-\mathrm{y}$ and $\mathrm{y}-\mathrm{z}$ plane.

fluence of $20 \mathrm{~mJ} \mathrm{~cm}^{-2}$. The reconstructed PA images of the arbitrary shaped phantoms are shown in the bottom panel of Figure 4 as $\mathrm{x}-\mathrm{y}$ and $\mathrm{x}-\mathrm{z}$ maximum intensity projections. The structure of the synthetic hair knot and twisted ribbons are accurately reproduced in the PA images. The intricate veins of the leaf skeleton phantom are also clearly visualized in the PA image.

\subsection{Ex ovo duck embryo}

Ex ovo duck embryos were used to further demonstrate the imaging capability of the system. Fourteen day-old duck embryos were imaged using an excitation wavelength of $590 \mathrm{~nm}$ and a fluence of $18 \mathrm{~mJ} \mathrm{~cm}{ }^{-2}$. Figure 5 shows the x-y maximum intensity projected PA images of the duck embryo, color coded according to the depth. The images show chorioallantoic membrane (CAM), which is the outermost extra-embryonic layer with dense blood vasculature. The fine network of blood vessels, some as small as $50 \mu \mathrm{m}$ across, in the CAM are clearly visualized in the PA images. 

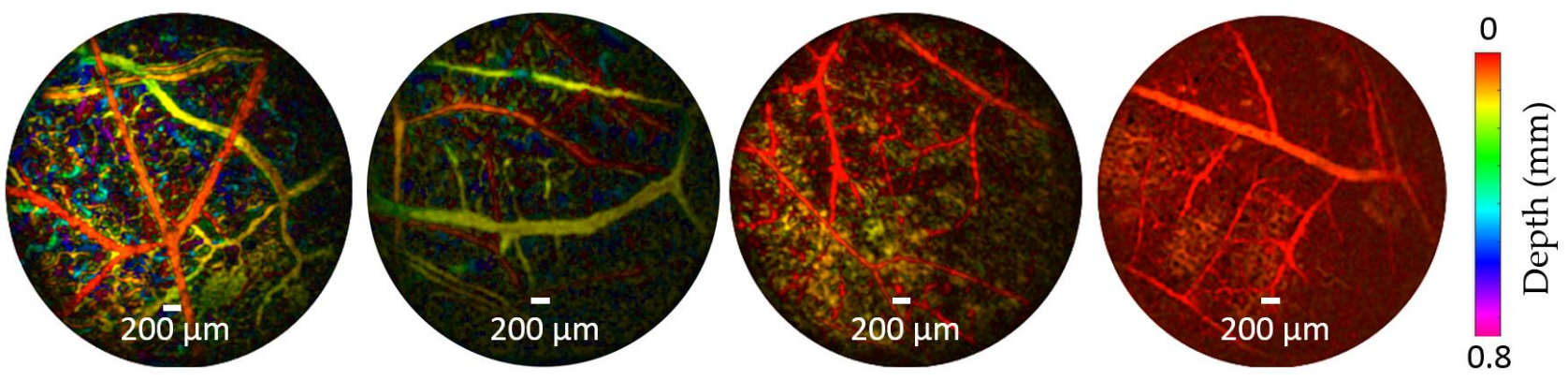

Figure 5. PA images (5 mm aperture) of chorioallontoic membrane (CAM) of the ex ovo duck embryo, acquired using an excitation wavelength of $590 \mathrm{~nm}$ and a fluence of $18 \mathrm{~mJ} \mathrm{~cm}^{-2}$. Images are colour-coded according to the depth.

\section{CONCLUSIONS}

The underlying principles of an all-optical, forward-viewing PAE probe for high resolution volumetric imaging have been demonstrated. This study has shown that it is possible to optically address the FP sensor using a $1.5 \mathrm{~mm}$ diameter flexible fibre bundle in order to synthesize a high-density 2D array of wideband ultrasound detectors at the distal end of the bundle. This allows for fine spatial-temporal sampling of the incident photoacoustic waves which results in high spatial resolution. The lateral spatial resolution of the probe is $50 \mu \mathrm{m}$ at a depth of $0.5 \mathrm{~mm}$ from the sensor surface and gradually decreases to $146 \mu \mathrm{m}$ at $6 \mathrm{~mm}$ in depth. The high-resolution imaging capability of the probe in biological tissue was demonstrated using ex ovo duck embryo, where the fine network of blood vessels in the chorion membrane were clearly visualized.

This new approach offers several advantages over previous distal-end scanning PAE probes such as an akinetic distal end, a forward-viewing configuration, relatively simple and inexpensive fabrication and the potential for wideband sensitive acoustic detection. It is also amenable to a high degree of miniaturization, as there are no active components at the distal end of the probe. To demonstrate proof-of-principle, standard bulk optical components were used at the distal end to interrogate the sensor. However, these could be miniaturized to reduce the outer diameter to mm-scale dimensions to allow for insertion into the working channel of standard endoscopes. Future work will focus on miniaturization of the distal end optics, optimization of acoustic sensitivity, faster image acquisition, and in vivo endoscopic imaging in animal models.

\section{ACKNOWLEDGMENTS}

This work was supported by an Innovative Engineering for Health award by the Wellcome Trust (No. WT101957) and the Engineering and Physical Sciences Research Council (EPSRC) (No. NS/A000027/1). The authors also acknowledge support from UK and European Union project FAMOS (FP7 ICT, Contract 317744).

\section{REFERENCES}

[1] Yang, J.-M., Maslov, K., Yang, H.-C., Zhou, Q., Shung, K. K., and Wang, L. V., "Photoacoustic endoscopy," Opt. Lett. 34, 1591-1593 (May 2009).

[2] Yang, J.-M., Favazza, C., Chen, R., Yao, J., Cai, X., Maslov, K., Zhou, Q., Shung, K. K., and Wang, L. V., "Simultaneous functional photoacoustic and ultrasonic endoscopy of internal organs in vivo," Nat. Med. 18, 1297-1303 (August 2012).

[3] Yang, J.-M., Chen, R., Favazza, C., Yao, J., Li, C., Hu, Z., Zhou, Q., Shung, K. K., and Wang, L. V., "A 2.5-mm diameter probe for photoacoustic and ultrasonic endoscopy," Opt. Express 20, 23944-23953 (Oct 2012).

[4] Dong, B., Chen, S., Zhang, Z., Sun, C., and Zhang, H. F., "Photoacoustic probe using a microring resonator ultrasonic sensor for endoscopic applications," Opt. Lett. 39(15), 4372-75 (2014).

[5] Yang, J. M., Favazza, C., Yao, J., Chen, R., Zhou, Q., Shung, K. K., and Wang, L. V., "Three-dimensional photoacoustic endoscopic imaging of the rabbit esophagus," PLOS ONE 10, 1-15 (04 2015). 
[6] Zhang, E., Laufer, J., and Beard, P., "Backward-mode multiwavelength photoacoustic scanner using a planar fabry-perot polymer film ultrasound sensor for high-resolution three-dimensional imaging of biological tissues," Appl. Opt. 47(4), 561-77 (2008).

[7] Zhang, E. Z., Laufer, J. G., Pedley, R. B., and Beard, P. C., "In vivo high-resolution 3d photoacoustic imaging of superficial vascular anatomy," Phys. Med. Biol. 54(4), 1035-46 (2009).

[8] Laufer, J., Norris, F., Cleary, J., Zhang, E., Treeby, B., Cox, B., Johnson, P., Scambler, P., Lythgoe, M., and Beard, P., "In vivo photoacoustic imaging of mouse embryos," J. Biomed. Opt. 17(6), 061220 (2012).

[9] Laufer, J., Johnson, P., Zhang, E., Treeby, B., Cox, B., Pedley, B., and Beard, P., "In vivo preclinical photoacoustic imaging of tumor vasculature development and therapy," J. Biomed. Opt. 17(5), 056016 (2012).

[10] Ansari, R., Mathews, S., Zhang, E. Z., Desjardins, A. E., and Beard, P. C., "Photoacoustic endoscopy probe using a coherent fibre optic bundle," Proc. SPIE 9539, Opto-Acoustic Methods and Applications in Biophotonics II 953905 (2015).

[11] Ansari, R., Zhang, E. Z., Desjardins, A. E., and Beard, P. C., "Photoacoustic endoscopy probe using a coherent fibre-optic bundle and fabry-prot ultrasound sensor," Proc. SPIE 9708, Photons Plus Ultrasound: Imaging and Sensing 97080L (2016).

[12] Beard, P. C., Perennes, F., and Mills, T. N., "Transduction mechanisms of the fabry-perot polymer film sensing concept for wideband ultrasound detection," IEEE Trans. Ultrason. Ferroelectr. Freq. Control 46, 157582 (1999).

[13] Treeby, B. E., Zhang, E., and Cox, B. T., "Photoacoustic tomography in absorbing acoustic media using time reversal," Inverse Problems 26, 115003 (2010). 